\title{
The Use of Near-Infrared Spectroscopy as a Substitute for Blood Pressure Monitoring in a Patient with Severe Osteogenesis Imperfecta
}

\author{
Joshua D. Dilley $^{1 *}$, Edwin J. Abraham ${ }^{2}$, Taranjit S. Sangari ${ }^{2}$ \\ ${ }^{1}$ Department of Anesthesiology, University of Arkansas for Medical Sciences, Little Rock, USA; ${ }^{2}$ Department of Pediatric Anesthe- \\ siology, Arkansas Children's Hospital, Little Rock, USA. \\ Email: jddilley@uams.edu
}

Received July $6^{\text {th }}$, 2012; revised August $6^{\text {th }}$, 2012; accepted August $26^{\text {th }}, 2012$

\begin{abstract}
The use of near-infrared spectroscopy (NIRS) as a means of assessing regional oxygen supply is a method that has gained recent support and interest. Given the potential of NIRS, this technology was utilized in an infant patient with a case of severe osteogenesis imperfecta that precluded conventional blood pressure monitoring. Using NIRS as a monitor and titrating the anesthetic accordingly produced a good outcome, with no post-operative evidence of detrimental intra-operative hypotension or ischemia.
\end{abstract}

Keywords: Near Infrared Spectroscopy; Osteogenesis Imperfecta; Monitoring

\section{Introduction}

The assessment of regional supply of Oxygen $\left(\mathrm{rSO}_{2}\right)$ via near-infrared spectroscopy (NIRS) has gained increasing interest amongst anesthesiologists over the past several years. NIRS and analysis of $\mathrm{rSO}_{2}$ has found much utility in cranial injuries, heart surgery, and carotid endarterectomies [1-3]. It has also been shown to minimize brain exposure to potential hypoxia in general surgeries as well [4]. Due to the value in potentially preventing cerebral hypoxia and the ability to provide cerebral and somatic oxygen saturation, NIRS was employed for an additional level of safety in a case where performance of blood pressure measurement was contrary to the well-being of the patient.

\section{Case Report}

The patient was a $5 \mathrm{~kg}$, 4-month-old, former 24 weeks preterm newborn, in the neonatal intensive care unit, who required Nissen fundoplication and gastric button placement secondary to reflux and chronic aspiration. His past medical history was significant for bilateral grade IV intraventricular hemorrhage, obstructive hydrocephalus, respiratory distress syndrome, bronchopulmonary dysplasia, osteogenesis imperfecta (with bilateral rib fractures and a right femur fracture, and a left humerus and ulnar fracture that were healed), newborn hemolytic disorder, neo-

${ }^{*}$ Corresponding author. natal jaundice, gastroesophageal reflux, and chronic aspiration. He had undergone a right ventricular subgaleal shunt and a ventriculoperitoneal shunt due to his hydrocephalus.

His lungs were clear and his heart rate was regular with no murmurs appreciated. His vital signs were within normal limits. He required $30 \% \mathrm{FiO}_{2}$ and was maintaining oxygen saturations in the upper 90 's and was at $97 \%$ on the morning of surgery. An arterial blood gas drawn three days prior revealed a $\mathrm{pH}$ of $7.36, \mathrm{PaCO}_{2}$ of 61 , $\mathrm{PaO}_{2}$ of 55, and a bicarbonate of 33.5. He was mildly anemic with a hemoglobin of 11.3. His labs, including a basic metabolic panel were otherwise within normal limits. A chest X-ray revealed stable atelectasis and bilateral rib fractures. A head CT revealed the presence of a right parietal shunt, right frontal extraventricular drain, and ventriculomegaly. An ECG and ECHO were performed which were within normal limits. He had a technetium scan that revealed multiple episodes of reflux.

During his stay in the neonatal intensive care unit, he had no blood pressures taken secondary to his fragile bones. An arterial line was placed for his previous procedures. Per the old anesthesia records, it was noted that the arterial line was difficult to place and required multiple attempts. As it was difficult to place the arterial line, and the likely need for future orthopedic surgeries, the decision was made not to place an arterial line for this case. 
The patient was transferred from NICU with 1 liter of oxygen delivered via nasal cannula during transport, with pulse oximetry, ekg and temperature monitors in place and were continued intraoperatively. A near-infrared spectroscopy monitor (Somanetics, Troy, Michigan, Model 5100 C. Probe Model RSC-1) was placed with probes on the child's forehead and back at the level of the left costovertebral angle. The probes were thin adhesive patches that were applied directly to the skin of the patient. A 24 gauge iv was in place in the left hand. General anesthesia was induced with $10 \mathrm{mg}$ of ketamine, $5 \mathrm{mcg}$ of fentanyl, and $5 \mathrm{mg}$ of rocuronium. Maintenance of anesthesia was achieved with inhaled sevoflurane.

Immediately after intubation, peak airway pressures in the 30 's $\mathrm{cm}$ of $\mathrm{H}_{2} \mathrm{O}$ range became necessary to adequately ventilate the patient. 8 puffs of albuterol was given via endotracheal tube, to which the patient responded. Airway pressures decreased to less than $20 \mathrm{~cm}$ of $\mathrm{H}_{2} \mathrm{O}$ with adequate ventilation, delivering $\sim 8 \mathrm{~mL} / \mathrm{kg}$ throughout the remainder of the case. Due to the patient's pulmonary history, a chest X-ray was taken at this time, in order to rule out any other complications. The X-ray revealed no acute changes. The ventilator settings were adjusted throughout the case to maintain an end tidal $\mathrm{CO}_{2}$ value near $40 \mathrm{mmHg}$. Pressure control mode was selected at a peak pressure of $29 \mathrm{mmHg}$ with a peep of 6 mmHG. The respiratory rate varied from an initial setting of $40 \mathrm{bpm}$ to a final rate of 50 .

Aside from the initial difficulties with ventilation, the case proceeded with no other complications. The patient showed no response to incision and received no more narcotics throughout the case. The NIRS monitor provided continuous data regarding the patient's regional oxygen saturation $\left(\mathrm{rSO}_{2}\right)$, a noninvasive indicator of organ perfusion. Prior to induction of anesthesia, a baseline was determined for the cerebral and somatic sites (74 \& 85 respectively). The current literature and manufacturer recommend that a normal cerebral $\mathrm{rSO}_{2}$ ranges between 58 - 82 points. Evidence supports intervention when the $\mathrm{rSO}_{2}$ decreases to 50 points or below, or a 20 point decrease from baseline. Any decrease greater that $25 \%$ or value less than 40 points is considered to be a critical change. The somatic $\mathrm{rSO}_{2}$ is generally 5 - 20 points higher than the cerebral value. The goal of the anesthetic was to follow these guidelines and titrate the fluid volume and depth of inhaled anesthetic to maintain the values within the aforementioned range. The cerebral $\mathrm{rSO}_{2}$ was maintained between 74 - 49 while the somatic $\mathrm{rSO}_{2}$ was maintained between 85 - 71 points (see Table 1).

The sevoflurane concentration was held constant at an end tidal value of $1.1 \%$. When the cerebral value dipped to 49 , a $5 \mathrm{~mL}$ bolus of crystalloid was given. The value initially trended upwards to the low 50's, then dipped to 50. At this time, another bolus of $5 \mathrm{~mL}$ was given, to which the $\mathrm{rSO}_{2}$ improved and remained in the upper 50's and was in the 60's at the end of the case. Pulse oximetry readings were constant at $100 \%$ after the initial difficulties at the beginning of the case. No changes occurred at the time of decreased $\mathrm{rSO}_{2}$. Had the patient not responded to the fluid bolus, one consideration would have been to increase the $\mathrm{FiO}_{2}$ to improve the $\mathrm{rSO}_{2}$.

The patient was transferred to the NICU with an endotracheal tube, an EKG and pulse oximetry monitors in place. He was extubated the following day. He remained in the hospital 2 weeks following the procedure and was discharged home. Although his age made it potentially difficult to assess, postoperatively, there were no signs or symptoms attributable to prolonged intraoperative hypotension. His neurological status and exam were unchanged from the preoperative period. He moved his head bilaterally. He tracked and followed objects with his eyes. He also had good upper and lower extremity tone bilaterally. He demonstrated no laboratory changes indicative of prolonged hypotension. His creatinine was 0.1 preoperatively and was postoperatively as well. A venous blood gas drawn revealed a $\mathrm{pH}$ of 7.32 which was slightly low, but unchanged from a gas drawn two days previously. The $\mathrm{pvCO}_{2}$ was relatively constant at 62.5 , increased slightly from $60.9 \mathrm{mmHG}$ on the previous draw. The $\mathrm{pvO}_{2}$ was also low at $39.9 \mathrm{mmHg}$. Secondary to his bronchopulmonary dysplasia, this value had been chronically low as well and had varied from 58.6 to 32.6.

\section{Discussion}

NIRS monitoring stems from the observation, first reported in 1977 [5], that brain and myocardial tissue are relatively transparent to light in the near-infrared range. The primary light absorbing molecules within the NIR range include hemoglobin, bilirubin, and cytochromes. As NIR light is delivered, there is a difference in intensity between the transmission through the skull and the reception of that light at a sensor. The decrease in intensityis proportional to amount of the hemoglobin present. Measurement of tissue oxygen saturation and hemoglo-

Table 1. NIRS $\mathrm{rSO}_{2}$ values.

\begin{tabular}{ccccccccc}
\hline Time & Baseline 0915 & 0930 & 0945 & 1000 & 1015 & 1030 & 1045 & 1100 \\
\hline Cerebral & 74 & 74 & 50 & 55 & 49 & 51 & 50 & 60 \\
Somatic & 85 & 85 & 84 & 71 & 83 & 80 & 83 & 83 \\
\hline
\end{tabular}


bin content is thus possible. The absorption of oxyhemoglobin occurs as a broad peak between 700 and $1150 \mathrm{~nm}$, whereas deoxyhemoglobin occurs from 650 to $1000 \mathrm{~nm}$. Wavelengths between 700 and $850 \mathrm{~nm}$ are typically employed in order to maximally differentiate between oxyand deoxyhemoglobin [6].

The noninvasive determination of the oxygen content of blood in critical organs clearly has significant potential and was utilized in this case. As blood pressure measurements were unable to be performed intraoperatively, the employment of NIRS in conjunction with the other vital signs provided a safely delivered anesthetic. The goal of the anesthetic was to maintain the cerebral and somatic $\mathrm{rSO}_{2}$ in the range suggested by the manufacturer described above. Depending on the specific situation encountered, options for modification included, decreasing anesthetic depth, fluid bolus, and modification of ventilator settings. In this case, the end tidal concentration of sevoflurane was maintained at $1.1 \%$ throughout, in order to maintain an appropriate anesthetic depth. Had the $\mathrm{rSO}_{2}$ not responded to the fluid bolus, the sevoflurane concentration would have been modified. Given the increase in heart rate, light anesthesia or pain was considered. Given the low $\mathrm{rSO}_{2}$, and positive response to fluid boluses, a decrease in blood pressure was felt to be more likely. Accordingly, the only narcotics that were given were done so during induction.

NIRS has been shown to be useful as an index of perfusion $[1,4,6]$. Based on this information, an assumption was made that evidence of adequate organ perfusion would suffice in lieu of a blood pressure reading. Despite the aforementioned studies, a major drawback of NIRS monitoring is the lack of large scale studies demonstrateing its utility. This is the first case reported where NIRS has been employed as a surrogate to blood pressure monitoring. This is not ideal as there is no evidence to support it being used as such. Clearly more studies need to be performed before the value of NIRS monitoring can be confidently known. Technical difficulties could have also provided misleading information during the case. Improper calibration and poor skin contact could have potentially resulted in the generation of incorrect values. Care must be taken to properly achieve a baseline value upon application and also to monitor the probe sites throughout the case.

Given the patient's age, the results of prolonged hypotension can be difficult to detect. The patient's neurological status was unchanged from the preoperative period. However, as the patient is unable to communicate, the physical exam must be relied upon exclusively. Subtle changes may go undetected given the low functionality and lack of fine motor skills of a child this young. The laboratory data, although important, is also not a specific marker of hypotension. Significant hypotension may have occurred throughout the case with unchanged values for creatinine and $\mathrm{pH}$ and other labs having occurred.

The surgical procedure performed during this case was one of both short duration and minimal fluid shifts. As such, the positive outcome obtained must not be accepted credulously. Standard monitors, as recommended by the American Society of Anesthesiologists, should be applied to patients undergoing any surgical procedure when- ever possible. As shown by this case, there are situations that exist that preclude utilization of all of the standard monitors. In such a situation, NIRS may be a useful substitute for blood pressure monitoring. One must keep in mind that the entire clinical picture should always be surveyed throughout the delivery of any anesthetic. All available data, not limited to pulse oximetry, heart rate, color, tone, palpable pulses, $\mathrm{FIO}_{2}$ and endtidal $\mathrm{CO}_{2}$, should be used together to generate an idea of the patient's overall well being in order to deliver the safest anesthetic possible.

\section{REFERENCES}

[1] A. Konishi and K. Kikuchi, "Cerebral Oxygen Saturation $\left(\mathrm{rSO}_{2}\right)$ during Open Heart Surgery and Postoperative Brain Dysfunction,” Masui, Vol. 44, No. 10, 1995, pp. 1322-1326.

[2] T. Higami, S. Kozawa, T. Asada, et al., "Retrograde Cerebral Perfusion versus Selective Cerebral Perfusion as Evaluated by Cerebral Oxygen Saturation during Aortic Arch Reconstruction," The Annals of Thoracic Surgery, Vol. 67, No. 4, 1999, pp. 1091-1096. doi:10.1016/S0003-4975(99)00135-6

[3] S. K. Samra, E. A. Dy, K. Welch, et al., "Evaluation of a Cerebral Oximeter as a Monitor of Cerebral Ischemia during Carotid Endarterectomy,” Anesthesiology, Vol. 93, No. 4, 2000, pp. 964-970. doi:10.1097/00000542-200010000-00015

[4] A. Casati, G. Fanelli, P. Pietropaoli, R. Proietti, R. Tufano, G. Danelli, G. Fierro, G. De Cosmo and G. Servillo, "Continuous Monitoring of Cerebral Oxygen Saturation in Elderly Patients Undergoing Major Abdominal Surgery Minimizes Brain Exposure to Potential Hypoxia,” Anesthesia \& Analgesia, Vol. 101, No. 3, 2005, pp. 740-747. doi:10.1213/01.ane.0000166974.96219.cd

[5] F. F. Jobsis, "Noninvasive, Infrared Monitoring of Cerebral and Myocardial Oxygen Sufficiency and Circulatory Parameters,” Science, Vol. 198, No. 4323, 1977, pp. 1264 1267. doi:10.1126/science.929199

[6] J. M. Murkin and M. Arango, "Near-Infrared Spectroscopy as an Index of Brain and Tissue Oxygenation,” British Journal of Anaesthesia, Vol. 103, No. 1, 2009, pp. i3i13. 